\title{
Integral Sliding Mode Control for Helicopter via Disturbance Observer and Quantum Information Technique
}

\author{
Qiang Qu, ${ }^{1}$ Fuyang Chen, ${ }^{1}$ Bin Jiang, ${ }^{1}$ and Gang Tao ${ }^{2}$ \\ ${ }^{1}$ College of Automation Engineering, Nanjing University of Aeronautics and Astronautics, Nanjing 210016, China \\ ${ }^{2}$ Department of Electrical and Computer Engineering, University of Virginia, Charlottesville, VA 22903, USA \\ Correspondence should be addressed to Qiang Qu; qq@nuaa.edu.cn
}

Received 6 August 2014; Accepted 19 September 2014

Academic Editor: Ke Zhang

Copyright (c) 2015 Qiang Qu et al. This is an open access article distributed under the Creative Commons Attribution License, which permits unrestricted use, distribution, and reproduction in any medium, provided the original work is properly cited.

\begin{abstract}
A novel self-repairing control scheme is proposed for a helicopter with unknown disturbance. Firstly, a disturbance observer is introduced to observe the disturbance of the system, which can produce corresponding control signals according to the disturbance signals. Secondly, an integral sliding mode controller is designed to compensate the unobserved disturbance and uncertainties. All of the closed-loop poles can be arbitrarily placed and the output errors converge to zero effectively through the controller. Besides, a robust closed-loop system against disturbance and parameter uncertainties is achieved. In addition, quantum information technique is used to increase the self-repairing control accuracy of helicopter. Finally, simulation results demonstrate the effectiveness and feasibility of the proposed self-repairing control scheme.
\end{abstract}

\section{Introduction}

A helicopter is a complicated aircraft and its performance is seriously influenced by environmental changes. Because of its nonlinearity, heavy coupling, varying parameters, and model uncertainty, a helicopter is very difficult to control. We can classify the characteristics of the helicopter into three categories: nonlinearity, uncertainty, and instability. The control of the helicopter, therefore, represents a challenge for control system design [1-3]. In recent years, lots of helicopter flight control schemes have been proposed, such as adaptive neural network control [4], fuzzy control [5], robust control [6], and adaptive back-stepping control.

Fuzzy control is a nonlinear control method, essentially. By using linguistic information, it possesses several advantages such as model-free, robustness, rule-based algorithm, and universal approximation theorem. It is also easy to use, simple to design, and strong in the anti-interference function. Recently, fuzzy control has been successfully and widely applied to many nonlinear systems [7-10].

Sliding mode control (SMC) is a robust method that is used to control nonlinear and uncertain systems. The SMC does not rely on an accurate aircraft mathematical model, and it can overcome model uncertainties and disturbance in the system, allowing the system track reference model with high precision. It also can stabilize some complex nonlinear systems which are difficult to be stabilized by the state feedback laws [11-15]. Integral sliding mode control (ISMC) is presented in [16], and it not only makes the uncertainties and disturbance rejected, but also achieves zero steady state error. Moreover, ISMC is more robust than conventional SMC in the application of electrohydraulic servo control systems.

Considering the existence of disturbance, quantum information technique is introduced in this paper. The study of quantum information technique $[17,18]$ has been a hot research topic; therefore, the scope of the applied research on quantum information technique is very wide $[19,20]$. In this paper, quantum information technique is used to increase the self-repairing control accuracy of the helicopter and improve the ability of anti-interference.

It is such a challenge to control a helicopter with its nonlinearity, heavy coupling, varying parameters, and model uncertainty. The main content of this paper is to design a direct self-repairing flight control system for the helicopter. The purpose of the system is to eliminate the influence of the parameter uncertainties and external disturbance. In this 
paper, an integrative method is proposed by combining fuzzy control, sliding mode control, and quantum information technique together, which therefore possesses the advantages of all three techniques. This method is both effective and feasible, which will be verified by simulation.

The proposed method can achieve the following superiorities.

(i) All of the closed-loop poles can be arbitrarily assigned.

(ii) The control system can be asymptotically stable and the output errors can converge to zero effectively.

(iii) Robust system [21, 22] against system parameter uncertainties and external disturbance can be achieved.

This paper not only theoretically proves the stability of the proposed method, but also verifies the effectiveness of this method through the simulation of the helicopter control system. Simulation results show that the proposed method provides a feasible method for the actual design of the helicopter control system.

This paper is organized as follows. Firstly, a disturbance observer is introduced to observe the disturbance of the system, which can produce control signals according to the disturbance signals. Secondly, ISMC with fuzzy tuning is designed. All of the closed-loop poles can be arbitrarily placed, and the design procedure includes the sliding function definition, the control law formulation, and the stability proof for the system. The sliding function involves the integral of the state as well as the output errors, so the output errors can converge to zero effectively. An additional fuzzy tuning control here is introduced to accelerate the reaching time and reduce chattering by utilizing fuzzy logic judge. Furthermore, quantum information technique is used to increase the self-repairing control accuracy of helicopter.

\section{Description of Mathematical Model}

The helicopter is a nonlinear system with strong coupling. In this paper, the linear model which is linearized about equilibrium point is only considered. The state equation of the model is as follows:

$$
\begin{gathered}
\dot{x}(t)=A x(t)+B u(t), \\
y(t)=C x(t),
\end{gathered}
$$

where $x(t) \in R^{n}$ and $y(t) \in R^{r}$ are the state and the output of the system, respectively. $u(t) \in R^{r}$ is the control vector. $A \in R^{n \times n}, B \in R^{n \times r}$, and $C \in R^{r \times n}$ are the parameter matrices.

Some assumptions have been made to achieve the method which is applied in the flight control system.

(1) The pair $(A, B)$ is completely controllable and $(A, C)$ is completely observable.

(2) The disturbance $n(t)$ is unknown and bounded.
(3) Denote $G_{i}$ as the $i$ th row of the matrix $G$ in (17). Let $\Gamma=\operatorname{diag}\left\{\gamma_{1}, \gamma_{2}, \ldots, \gamma_{r}\right\}$, where $\gamma_{i}>0, i=1, \ldots$, $r$. For the disturbance $n(t, x)=\left[n_{1}(t, x), n_{2}(t, x), \ldots\right.$, $\left.n_{n}(t, x)\right]^{\mathrm{T}}$, the following conditions are satisfied: $\left\|G_{i} n(t, x)\right\| \leq \gamma_{i}\|x\|, i=1, \ldots, r$.

\section{Quantum Information Technique}

Quantum information is a new discipline, which is combined by quantum mechanics and information science. In recent years, based on the incomparable advantages on data transmission security, sensor measurement sensitivity and accuracy, and quantum computing parallelism, it has attracted widespread attention and development. Currently, the central issue of quantum information technique applied research is quantum cryptography, quantum communication, quantum computation, quantum simulation, quantum metrology, the physical basis of quantum information, and so on. By using quantum information technique, the researchers can simplify the modeling pattern so that the problems would be much easier.

In quantum computation, $|0\rangle$ and $|1\rangle$ denote the two basic states of microparticles, which are named as quantum bit (qubit). Arbitrary single-qubit state can be expressed as the linear combination of two basic states. The state of qubit is not only $|0\rangle$ and $|1\rangle$, but also a linear combination of the state, usually called as superposition state; namely,

$$
|\varphi\rangle=\alpha \cdot|0\rangle+\beta \cdot|1\rangle,
$$

where $\alpha$ and $\beta$ are a pair of complex, called the probability amplitude of quantum state; namely, the measurement results in quantum state $|\varphi\rangle$ collapsing $|0\rangle$ with a probability of $|\alpha|^{2}$ or collapsing $|1\rangle$ with a probability of $|\beta|^{2}$ and satisfying

$$
|\alpha|^{2}+|\beta|^{2}=1
$$

Therefore, quantum state can be also denoted by the probability amplitude of quantum state in the form of $|\varphi\rangle=[\alpha, \beta]^{\mathrm{T}}$. Obviously, if $\alpha=1, \beta=0,|\varphi\rangle$ is the basic state $|0\rangle$, which can be described by $|\varphi\rangle=[1,0]^{\mathrm{T}}$, then $\alpha=0, \beta=1,|\varphi\rangle$ is the basic state $|1\rangle$, which can be described by $|\varphi\rangle=[0,1]^{\mathrm{T}}$. Generally speaking, quantum state is the unit vector of twodimensional complex vector space.

Due to the collapse of quantum states caused by observation, there is a continuous state between the quantum bits $|0\rangle$ and $|1\rangle$, until it has been observed. The existence of continuous state qubit and behavior has been confirmed by a large number of experiments. And there are many different physical systems that can be used to realize quantum bits.

Similar to the single-qubit state, double-quantum-bit state can be expressed as

$$
|\varphi\rangle=\alpha_{00} \cdot|00\rangle+\alpha_{01} \cdot|01\rangle+\alpha_{10} \cdot|10\rangle+\alpha_{11} \cdot|11\rangle
$$

with the probability amplitude satisfying

$$
\left|\alpha_{00}\right|^{2}+\left|\alpha_{01}\right|^{2}+\left|\alpha_{10}\right|^{2}+\left|\alpha_{11}\right|^{2}=1 \text {. }
$$


Similarly, three-qubit state can be expressed as

$$
\begin{aligned}
|\phi\rangle= & \alpha_{000} \cdot|000\rangle+\alpha_{001} \cdot|001\rangle \\
& +\alpha_{010} \cdot|010\rangle+\alpha_{011} \cdot|011\rangle \\
& +\alpha_{100} \cdot|100\rangle+\alpha_{101} \cdot|101\rangle \\
& +\alpha_{110} \cdot|110\rangle+\alpha_{111} \cdot|111\rangle
\end{aligned}
$$

And the probability amplitude satisfying

$$
\begin{aligned}
& \left|\alpha_{000}\right|^{2}+\left|\alpha_{001}\right|^{2}+\left|\alpha_{010}\right|^{2}+\left|\alpha_{011}\right|^{2} \\
& \quad+\left|\alpha_{100}\right|^{2}+\left|\alpha_{101}\right|^{2}+\left|\alpha_{110}\right|^{2}+\left|\alpha_{111}\right|^{2}=1 .
\end{aligned}
$$

To increase the self-repairing control accuracy of helicopter, quantum information technique is added in the approach. The probability amplitudes of two quantum bits for the module can be seen in Table 1 .

\section{Disturbance Observer}

The helicopter is more or less subjected to disturbance, and it will affect the system performance. Hence, the disturbance observer [23] is designed so that the disturbance can be suppressed. The structure of the system is expressed as Figure 1.

Suppose the system has subjected to unknown external disturbance, the helicopter can be expressed as the following form:

$$
\begin{gathered}
\dot{x}(t)=A x(t)+B u(t)+n(t), \\
y(t)=C x(t),
\end{gathered}
$$

where $n(t) \in R^{n}$ is the external disturbance. Let $\widehat{n}$ be the value estimated of the disturbance and define:

$$
\widetilde{n}=n-\widehat{n}
$$

to be the observed error of the observer. $L(x)$ is the gain of the observer and $p(x)$ is a function that needs to be designed.

Then, we obtain the form of the disturbance observer as follows:

$$
\begin{gathered}
\widehat{n}=z+p(x), \\
\dot{z}=-L(x)+L(x) \cdot[-p(x)-A(x)-B u],
\end{gathered}
$$

where $L(x)$ and $p(x)$ should satisfy the following equation:

$$
L(x) \dot{x}=\frac{d p(x)}{d t} .
$$

The error dynamic of the observer can be expressed as

$$
\begin{aligned}
\dot{\tilde{n}} & =\dot{n}-\dot{\widehat{n}}=-\dot{z}-\dot{p} \\
& =L(z+p)-L(\dot{x}-A x-B u) \\
& =L \widehat{n}-L n=-L \tilde{n} .
\end{aligned}
$$

TABLE 1: Two quantum bit probability amplitude for quantum control module.

\begin{tabular}{lcc}
\hline Probability amplitude & Input & Output \\
& Yes $\backslash$ no & Yes \no \\
\hline$\alpha_{00}$ & No & No \\
$\alpha_{01}$ & No & Yes \\
$\alpha_{10}$ & Yes & No \\
$\alpha_{11}$ & Yes & Yes \\
\hline
\end{tabular}

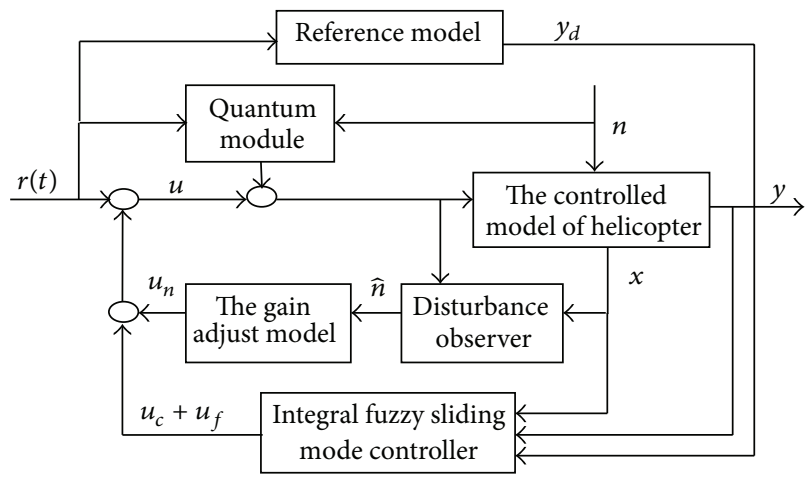

FIGURE 1: Integral sliding mode control for the helicopter via quantum information techniques and disturbance observer.

Select $L(x)=b, b>0$, and $p(x)$ is then designed as follows:

$$
p(x)=b x
$$

and then the observed error of the disturbance observer will be exponential convergence. The output of the observer is then converted into the corresponding input signal, which is expressed as

$$
u_{n}=B^{-1} \widehat{n} .
$$

Remark 1. The disturbance observer is introduced to observe the disturbance of the system, which can produce control signals according to the disturbance signals. By selecting the appropriate design parameters, the disturbance observer can be exponential convergence. The disturbance observer can reduce the burden of the controller, and the unobserved disturbance can be compensated by the ISMC. The simulation results verify that the disturbance observer can improve the tracking accuracy of the corresponding output.

\section{Integral Sliding Mode Control with Fuzzy Tuning}

The purpose here is to place all of the closed-loop poles and to eliminate the steady state errors.

The control function can be expressed as

$$
u=u_{c}+u_{f}
$$

where $u_{c}$ represents the ISMC and $u_{f}$ represents the fuzzy tuning control. 
Reference output $y_{d}=\left[y_{1 d}, y_{2 d}, \ldots, y_{r d}\right]^{\mathrm{T}}$, which is generated from the reference model, and define output error as $e=y-y_{d}$. The sliding function $\sigma$ is defined as

$$
\begin{aligned}
\sigma & =\left[\sigma_{1}, \sigma_{2}, \ldots, \sigma_{r}\right]^{\mathrm{T}}=-G x+H \int_{0}^{t} e d \tau \\
& =-G x+H \int_{0}^{t}\left(y-y_{d}\right) d \tau,
\end{aligned}
$$

where the matrix $H \in R^{r \times r}$ is selected to be full ranked and $G \in R^{r \times n}$ is selected such that $G B$ is nonsingular.

Theorem 2. Consider the system in (2) and (3) and the sliding function defined in (16). G can be selected as

$$
G=H C A_{d}{ }^{-1}
$$

where the matrix $A_{d} \in R^{n \times n}$ is the desired nonsingular closedloop system matrix. Then, in the sliding mode, the closed-loop poles are exactly the eigenvalues of the desired system matrix $A_{d}$.

Proof of the theorem is as [13].

In the sliding mode, the linear equivalent control is generated by setting $\dot{\sigma}=0$, which yields

$$
u_{\mathrm{eq}}=-(G B)^{-1}(G A x-H e+G n) .
$$

The control law be designed and expressed as

$$
u_{c}=\Lambda x+\Psi e+\Phi \operatorname{sgn}(\sigma) \text {. }
$$

To satisfy the sliding condition,

$$
\sigma_{i} \dot{\sigma}_{i}<0
$$

where

$$
\begin{gathered}
\Lambda=-(G B)^{-1} G A, \\
\Psi=(G B)^{-1} H, \\
\Phi=(G B)^{-1} \Gamma\|x\| .
\end{gathered}
$$

By taking the derivative of both sides of (16) and applying it to (19)-(21), we can get

$$
\dot{\sigma}=-\Gamma\|x\| \operatorname{sgn}(\sigma)-G n
$$

for $i=1, \ldots, i$ :

$$
\begin{aligned}
\dot{\sigma}_{i} & =-\gamma_{i}\|x\| \operatorname{sgn}\left(\sigma_{i}\right)-G_{i} n \\
& \leq-\left(\gamma_{i}\|x\|-\left\|G_{i} n\right\| \operatorname{sgn}\left(\sigma_{i}\right)\right) \operatorname{sgn}\left(\sigma_{i}\right) .
\end{aligned}
$$

Because the sum of the terms in the above brackets is larger than zero, the sliding condition (20) is therefore satisfied with the sliding function definition (16) and the control law (19). The control law given by (19) guarantees that the sliding model will be reached and sustained.
As we know, the discontinuous switching control signal $\operatorname{sgn}(s)$ often leads to chattering, which excites undesired highfrequency unmodeled dynamics. An additional fuzzy tuning control [24] here is introduced to accelerate the reaching time and to reduce chattering by utilizing fuzzy logic judge. follows:

The fuzzy rules for continuously adjusting $u_{f}$ are as if $\sigma$ is negative large, then $u_{f}$ is positive large; if $\sigma$ is negative small, then $u_{f}$ is positive small; if $\sigma$ is positive large, then $u_{f}$ is negative large; if $\sigma$ is positive small, then $u_{f}$ is negative small.

The sigmoidal membership functions are chosen as

$$
\begin{gathered}
\mu_{\sigma \text {-large }}=\frac{\exp (\sigma / \lambda)-\exp (-\sigma / \lambda)}{\exp (\sigma / \lambda)+\exp (-\sigma / \lambda)} \\
\mu_{\sigma \text {-small }}=1-\mu_{\sigma \text {-large }}
\end{gathered}
$$

where $\lambda$ is a positive constant.

The singletons chosen as membership functions for $u_{f}$ are as follows:

$$
\begin{aligned}
& \mu_{u_{f} \text {-large }}= \begin{cases}1, & u_{f}=-v, \\
0, & u_{f} \neq-v,\end{cases} \\
& \mu_{u_{f} \text {-small }}= \begin{cases}1, & u_{f}=0, \\
0, & u_{f} \neq 0,\end{cases}
\end{aligned}
$$

where $v$ is a scaling constant.

When $s>0$, we get the fuzzy tuning control $u_{f}$ as follows:

$$
\begin{aligned}
u_{f} & =\frac{\mu_{\sigma \text {-large }} \cdot(-\nu)+\mu_{\sigma \text {-small }} \cdot(0)}{\mu_{\sigma \text {-large }}+\mu_{\sigma \text {-small }}} \\
& =-\nu \mu_{\sigma \text {-large }} .
\end{aligned}
$$

Similarly, we can get the same expression for the fuzzy tuning control $u_{f}$ when $s<0$.

Remark 3. The resulting control system has the following advantages:

(1) all of the closed-loop poles can be arbitrarily placed;

(2) the output errors can converge to zero effectively;

(3) the system performance is robust.

\section{Simulation Analysis}

In this section, an example of a helicopter model is given to demonstrate the effectiveness and feasibility of the proposed control approach. Considering the helicopter system described by the form of (1), where $x(t)=[u, w, q, \theta]^{\mathrm{T}}$ is the state variable, $u, w, q$, and $\theta$ are the horizontal component, the vertical component, the pitch rate, and the pitch angle, respectively. $u(t)=\left[u_{\text {col }}, u_{\text {lon }}\right]^{\mathrm{T}}$ is the control vector, and its 


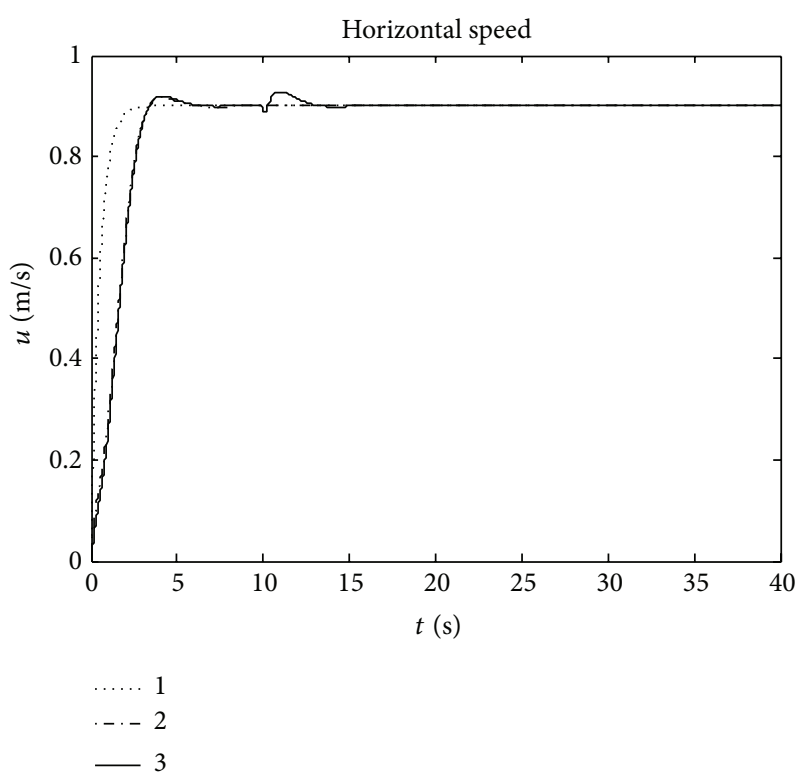

(a) Horizontal speed of the helicopter

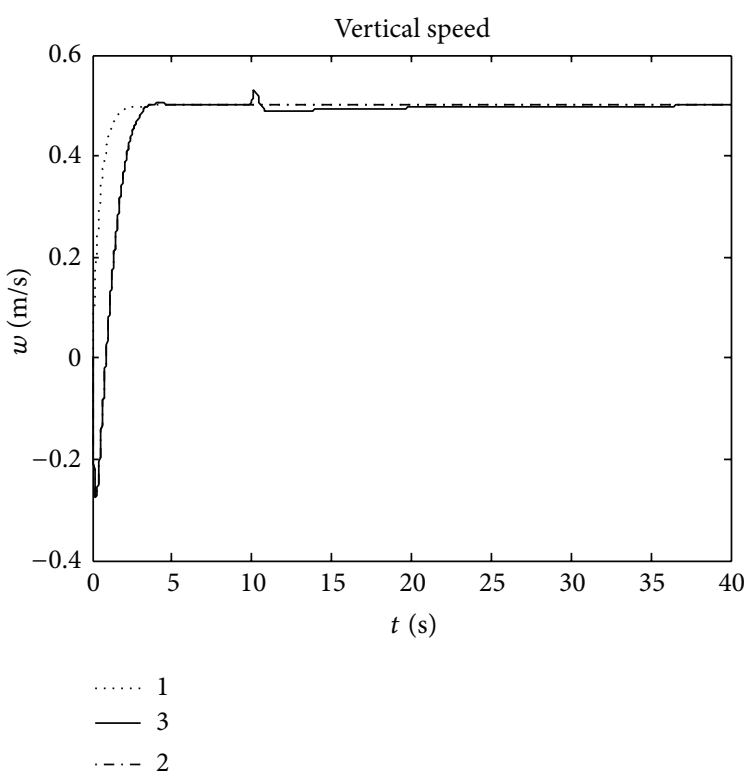

(b) Vertical speed of the helicopter

FIGURE 2: Control performance of the helicopter.

variables are the total distance variable and the longitudinal periodic variable. $y=[u, w]^{\mathrm{T}}$ is the output vector. Consider

$$
\begin{gathered}
A=\left[\begin{array}{cccc}
-0.036 & 0.0271 & 0.0188 & -0.4555 \\
0.0482 & -1.010 & 0.0024 & -4.0208 \\
0.1002 & 0.06635 & -0.7070 & 0.1198 \\
0 & 0 & 1 & 0
\end{array}\right], \\
B=\left[\begin{array}{cc}
0.4422 & 0.1761 \\
0.9775 & -7.5922 \\
-5.522 & 4.490 \\
0 & 0
\end{array}\right], \quad C=\left[\begin{array}{llll}
1 & 0 & 0 & 0 \\
0 & 1 & 0 & 0
\end{array}\right] .
\end{gathered}
$$

The control system is unstable by analysis. So to make the control system stable and obtain better dynamic performance, we assume that the desired closed-loop pole is $P=$ $[-1+i,-1-i,-5,-30]$, and then we can get the feedback matrix:

$$
K=\left[\begin{array}{cccc}
-7.5146 & -0.1165 & 2.333 & 4.794 \\
3.89 & -4.7539 & -0.6862 & -2.035 \\
76.3833 & 4.7302 & -24.7311 & -57.3561 \\
0 & 0 & 1 & 0
\end{array}\right]
$$

The desired system matrix is

$$
A_{d}=A-B K \text {. }
$$

Then, we design the sliding surface. Assume the weighting matrix $H$ is a unit matrix, and then according to (11) and (15)(17), we can get the following matrices:

$$
\begin{aligned}
G & =\left[\begin{array}{cccc}
-0.9404 & -0.0533 & -0.0767 & 0.2602 \\
-0.2254 & -0.216 & -0.0112 & 0.1012
\end{array}\right], \\
\Lambda & =\left[\begin{array}{cccc}
0.3803 & 0.6124 & 4.9437 & 11.3918 \\
0.0633 & -0.0379 & 0.7272 & 1.2042
\end{array}\right],
\end{aligned}
$$

$$
\begin{gathered}
\Psi=\left[\begin{array}{cc}
-16.276 & -1.1081 \\
-2.6159 & 0.4672
\end{array}\right] \\
\Phi=\left[\begin{array}{cc}
-16.276 & -1.1081 \\
-2.6159 & 0.4672
\end{array}\right]\left[\begin{array}{l}
0.005 \\
0.001
\end{array}\right]\|x\| .
\end{gathered}
$$

And the disturbance $n(t)$ is as follows:

$$
n(t)= \begin{cases}{[0,0,0,0]^{\mathrm{T}},} & 0 \leq t<10, \\ {[0.5,1,0,0]^{\mathrm{T}},} & 10 \leq t<40 .\end{cases}
$$

The responses of the horizontal speed and vertical speed are shown in Figures 2 and 3. The reference input is $r(t)=$ $[5 ; 5]$. The disturbance is supposed to occur at $t=10 \mathrm{~s}$.

In Figure 2, curve 1 indicates the desired output; curve 2 indicates the output using the proposed method without disturbance; and curve 3 indicates the output using the proposed method with disturbance. From the simulation results, we can see that the control system has a stronger selfrepairing and anti-interference capability.

In Figure 3, curve 1 indicates the desired output; curve 2 indicates the output using the ISMC with fuzzy tuning via quantum information techniques and disturbance observer proposed in this paper; curve 3 indicates the output using the ISMC with fuzzy tuning; and curve 4 indicates the output using the ISMC.

From the simulation results, we can see that the control quality of the helicopter flight control system using the ISMC is not so good. There are steady output errors; that is, the method cannot make sure that the outputs of the controlled system follow the desired outputs. With the ISMC with fuzzy tuning, the helicopter control system can track the desired outputs. With the proposed approach, the output tracking is 


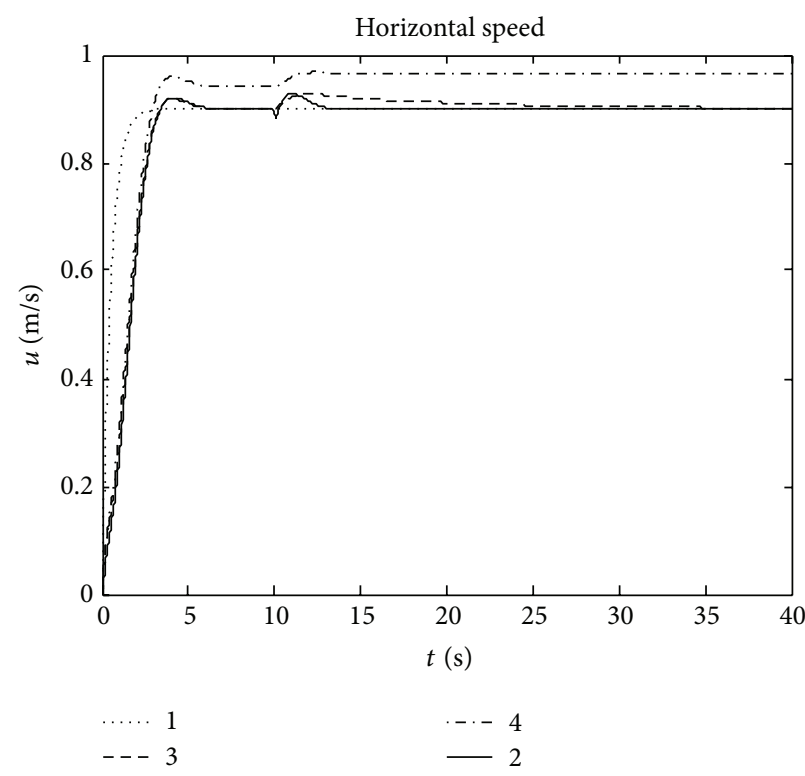

(a) Horizontal speed

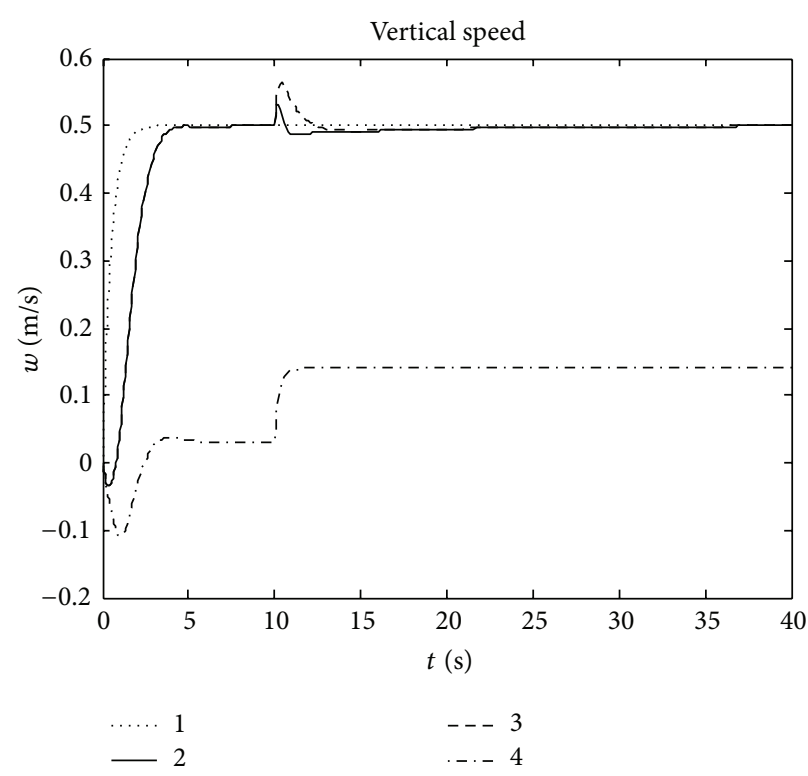

(b) Vertical speed

FIGURE 3: Control performance of the horizontal speed and vertical speed.

fast and accurate. When the disturbances occur, the system has the ability to drive back to the desired path quickly and the steady output errors converge to zero. It has stronger selfrepairing and anti-interference capabilities.

In order to make full validation of the proposed method, the method has been applied to semiphysical simulation platform of the actual flight control system. Compared with the above mathematical simulation, semiphysical simulation is closer to the actual system, and it can more fully reflect the helicopter flight process. Semiphysical simulation requires higher accuracy, reliability, and real time [25].

The 3-DOF helicopter is shown in Figure 4. It mainly consists of helicopter body, base, position sensors, propeller motors, balance block, and slip ring. The base is as the fulcrum to the balance bar. Propeller and balance blocks are installed at both ends of the balance bar. The lift force generated by the propeller can make the balance bar do pitching movements. The speed difference generated by the two propellers can make the balance bar do rotating movements.

The semiphysical simulation results verify that the 3-DOF helicopter can run safely with disturbance using the method proposed in this paper. By analyzing the simulation results, we can find that they meet the technical requirements of the flying qualities. The proposed method has the practical significance.

\section{Conclusion}

In this paper, a self-repairing control scheme is proposed for the helicopter flight control system with unknown disturbance. The process of the disturbance observer design and the ISMC with fuzzy tuning are introduced. In addition, quantum

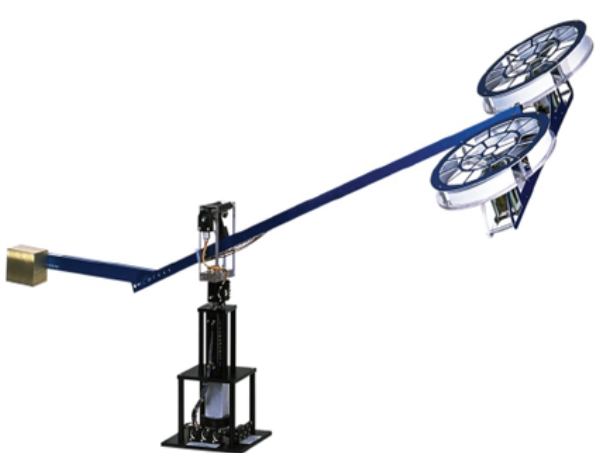

FIGURE 4: The 3-DOF helicopter system.

information technique is briefly introduced, which is used to increase the self-repairing control accuracy of helicopter and improve the ability of anti-interference. Simulation results show that the proposed method provides an effective and feasible approach for the actual design of the helicopter control system.

\section{Disclosure}

This paper has never been submitted to any journal or conference.

\section{Conflict of Interests}

The authors declare that there is no conflict of interests regarding the publication of this paper. 


\section{Acknowledgments}

The project was supported by the Aeronautics Science Foundation of China (2014ZC52033) and the National Natural Science Foundation of China $(61374130,61473146)$.

\section{References}

[1] I. A. Raptis, K. P. Valavanis, and G. J. Vachtsevanos, "Linear tracking control for small-scale unmanned helicopters," IEEE Transactions on Control Systems Technology, vol. 20, no. 4, pp. 995-1010, 2012.

[2] P. E. I. Pounds, D. R. Bersak, and A. M. Dollar, "Stability of small-scale UAV helicopters and quadrotors with added payload mass under PID control," Autonomous Robots, vol. 33, no. 1-2, pp. 129-142, 2012.

[3] W. Wang, K. Nonami, and Y. Ohira, "Model reference sliding mode control of small helicopter X.R.B based on vision," International Journal of Advanced Robotic Systems, vol. 5, no. 3, pp. 235-242, 2008.

[4] P. T. Keng, S. G. Shuzhi, and F. E. H. Tay, "Adaptive neural network control for helicopters in vertical flight," IEEE Transactions on Control Systems Technology, vol. 16, no. 4, pp. 753-762, 2008.

[5] P. Quiñones-Reyes, J. Ortega-Arjona, E. Méndez-Monroy, H. Benítez-Pérez, and A. Durán-Chavesti, "Fuzzy control design for a class of nonlinear network control system: helicopter case study," International Journal of Computers, Communications \& Control, vol. 7, no. 2, pp. 365-376, 2012.

[6] H.-C. Kim, H. R. Dharmayanda, T. Kang, A. Budiyono, G. Lee, and W. Adiprawita, "Parameter identification and design of a robust attitude controller using $H_{\infty}$ methodology for the raptor E620 small-scale helicopter," International Journal of Control, Automation and Systems, vol. 10, no. 1, pp. 88-101, 2012.

[7] J. T. Fei, W. R. Juan, and T. H. Li, "An adaptive fuzzy control approach for the robust tracking of a MEMS gyroscope sensor," International Journal of Advanced Robotic Systems, vol. 8, no. 5, pp. 125-133, 2011.

[8] F. M. Raimondi and M. Melluso, "Fuzzy/Kalman hierarchical horizontal motion control of underactuated ROVs," International Journal of Advanced Robotic Systems, vol. 7, no. 2, pp. 139$154,2010$.

[9] C. C. Lee, "Fuzzy logic in control systems: fuzzy logic controller. Part I/II," Institute of Electrical and Electronics Engineers. Transactions on Systems, Man, and Cybernetics, vol. 20, no. 2, pp. 419435, 1990.

[10] A. Boulkroune, M. M'Saad, and M. Farza, "Adaptive fuzzy tracking control for a class of MIMO nonaffine uncertain systems," Neurocomputing, vol. 93, pp. 48-55, 2012.

[11] B. Zi, H. Sun, Z. Zhu, and S. Qian, "The dynamics and sliding mode control of multiple cooperative welding robot manipulators," International Journal of Advanced Robotic Systems, vol. 9, article 53, 2012.

[12] Y. Shtessel, J. Buffington, and S. Banda, "Tailless aircraft flight control using multiple time scale reconfigurable sliding modes," IEEE Transactions on Control Systems Technology, vol. 10, no. 2, pp. 288-296, 2002.

[13] Y. J. Huang, T. C. Kuo, and H. K. Way, "Robust vertical takeoff and landing aircraft control via integral sliding mode," IEE Proceedings: Control Theory and Applications, vol. 150, no. 4, pp. 383-388, 2003.
[14] T. Chatchanayuenyong and M. Parnichkun, “Time optimal hybrid sliding mode-PI control for an autonomous underwater robot," International Journal of Advanced Robotic Systems, vol. 5, no. 1, pp. 91-98, 2008.

[15] X. H. Yu and O. Kaynak, "Sliding-mode control with soft computing: a survey," IEEE Transactions on Industrial Electronics, vol. 56, no. 9, pp. 3275-3285, 2009.

[16] D. S. Yoo and M. J. Chung, "A variable structure control with simple adaptation laws for upper bounds on the norm of the uncertainties," IEEE Transaction on Automatic Control, vol. 37, no. 6, pp. 860-865, 1992.

[17] Z. H. Chen, D. Y. Dong, and C. B. Zhang, Quantum Control Theory, University of Science and Technology of China Press, Hefei, China, 2005.

[18] S. Cong, "Quantum system control survey of progress in quantum control system," Chinese Journal of Quantum Electronica, vol. 20, no. 1, pp. 1-9, 2003.

[19] F. Y. Chen, B. Jiang, and Y. Y. Duan, "Fault self-repairing flight control of a small helicopter via quantum control techniques," in Proceedings of the International Bhurban Conference on Applied Sciences and Technology, pp. 184-188, Islamabad, Pakistan, 2011.

[20] D. Y. Dong, C. L. Chen, T.-J. Tarn, A. Pechen, and H. Rabitz, "Incoherent control of quantum systems with wavefunctioncontrollable subspaces via quantum reinforcement learning," IEEE Transactions on Systems, Man, and Cybernetics, Part B: Cybernetics, vol. 38, no. 4, pp. 957-962, 2008.

[21] M. A. Trujano, R. Garrido, and A. Soria, "Robust visual control of parallel robots under uncertain camera orientation," International Journal of Advanced Robotic Systems, vol. 9, article 106, 2012.

[22] W. Siwakosit, S. A. Snell, and R. A. Hess, "Robust flight control design with handling qualities constraints using scheduled linear dynamic inversion and loop-shaping," IEEE Transactions on Control Systems Technology, vol. 8, no. 3, pp. 483-494, 2000.

[23] X. S. Liu, Y. Q. Wu, G. Q. Shi, and M. L. Hu, "Design of quadratic optimal controller based on disturbance observer," Journal of Gun Launch \& Control, vol. 4, pp. 47-51, 2007.

[24] Q. P. Ha, "Robust sliding mode controller with fuzzy tuning," Electronics Letters, vol. 32, no. 17, pp. 1626-1628, 1996.

[25] Q. J. Zhang, L. Liu, J. F. Zhang, A. J. Li, and X. Yu, "Design of hardware in the loop simulation system for a helicopter," Computer Measurement \& Control, vol. 14, no. 3, pp. 400-403, 2006. 


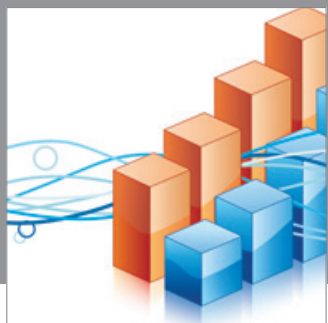

Advances in

Operations Research

mansans

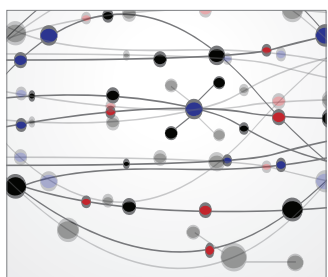

The Scientific World Journal
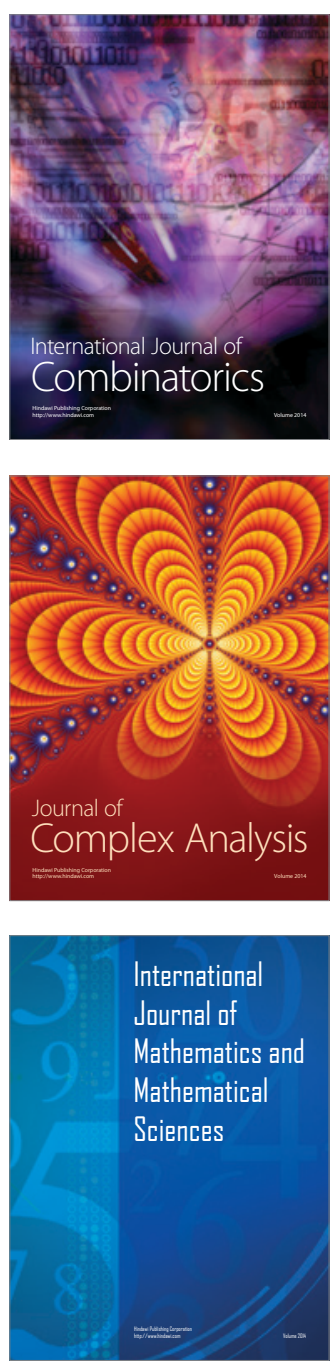
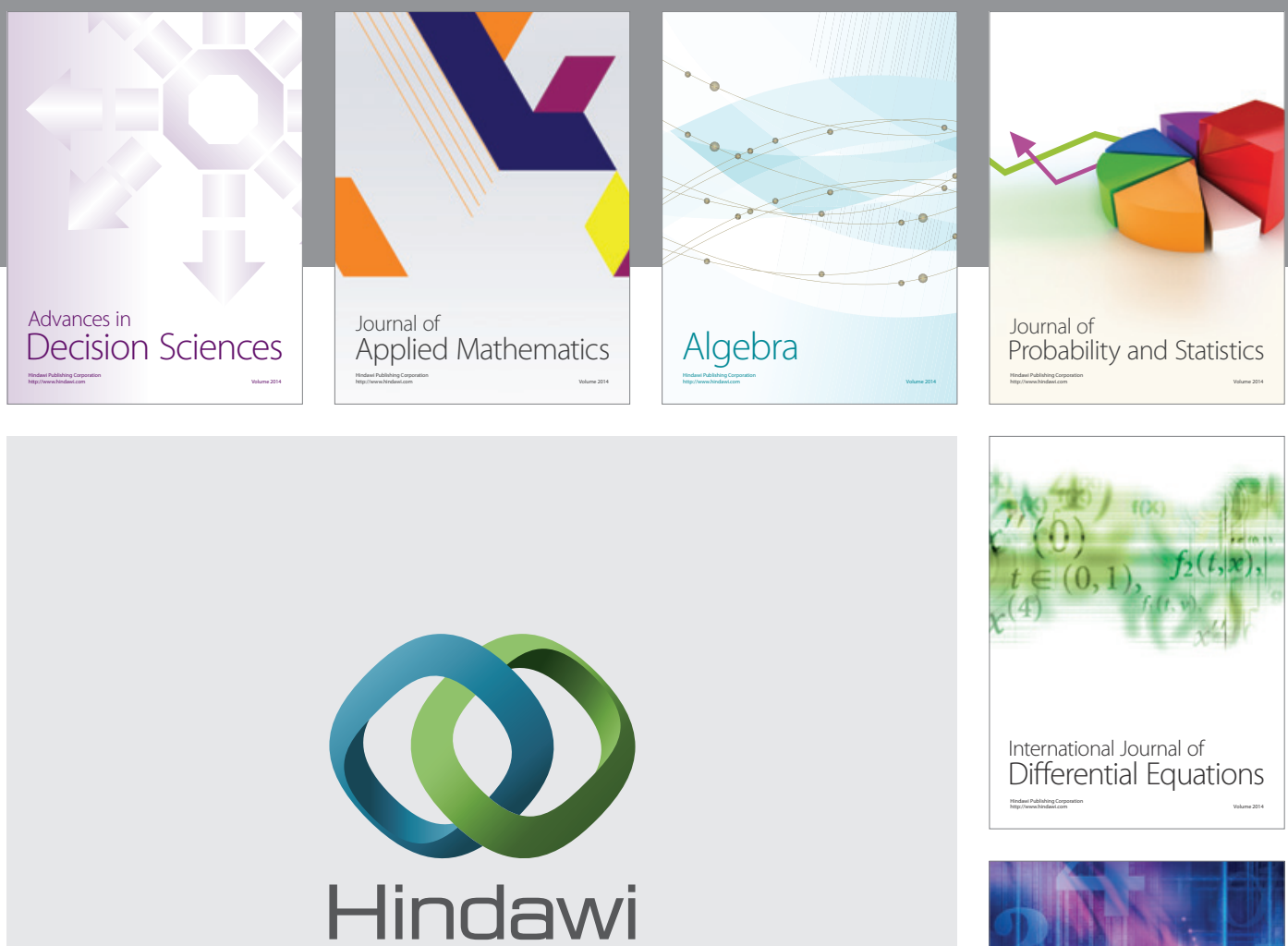

Submit your manuscripts at http://www.hindawi.com
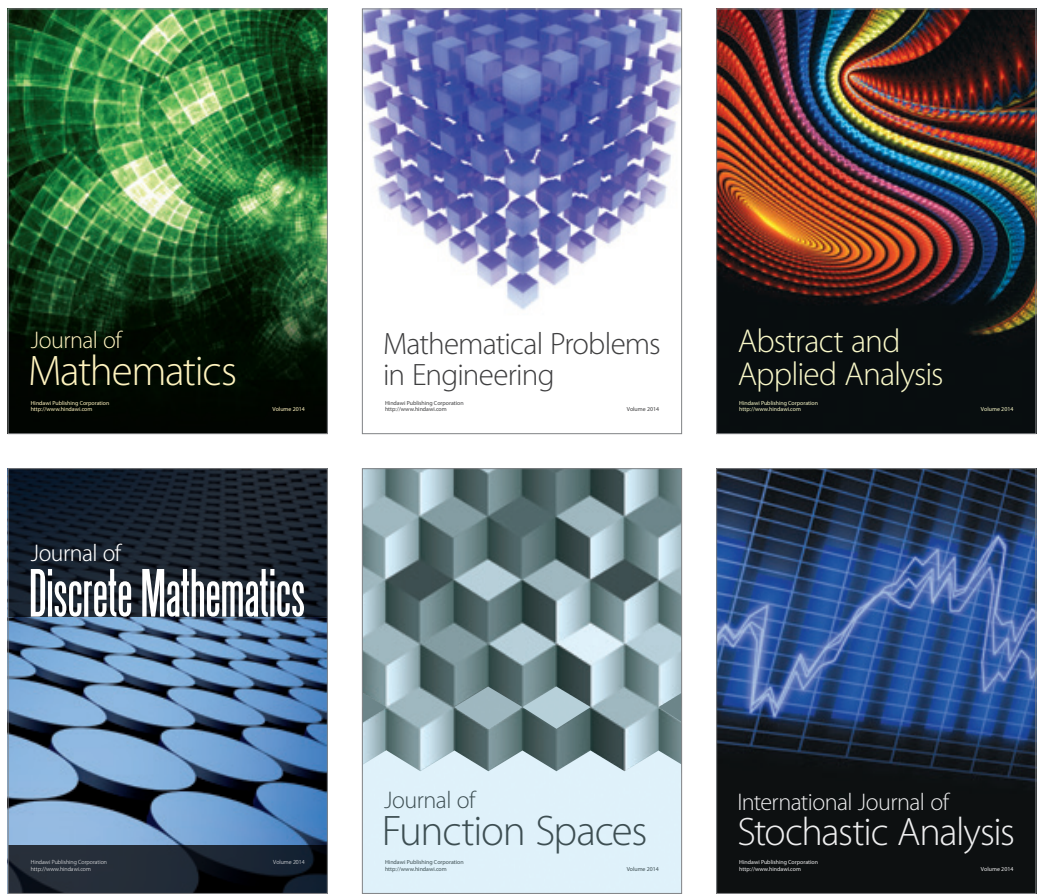

Journal of

Function Spaces

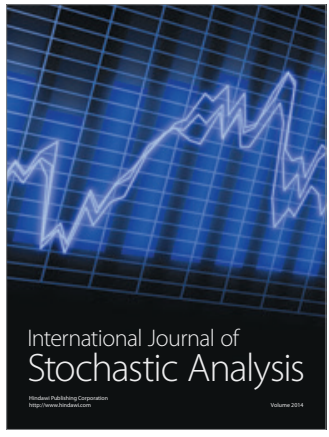

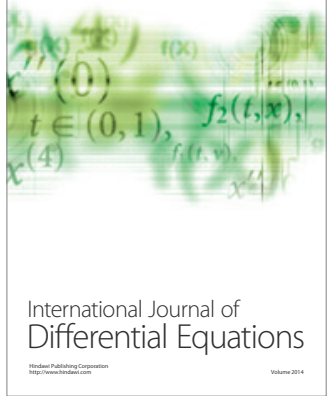
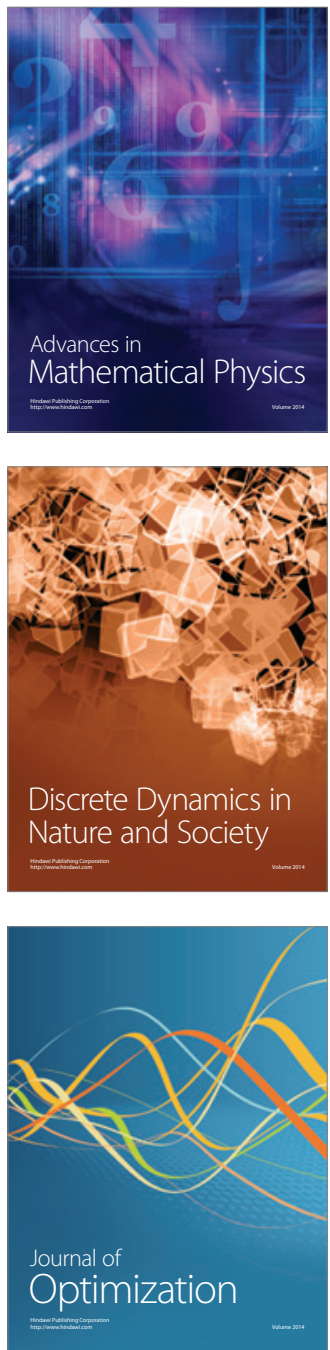